\title{
Molecular typing divides marine mammal strains of Brucella into at least three groups with distinct host preferences \\ Correspondence \\ Adrian M. Whatmore \\ a.whatmore@vla.defra.gsi.gov.uk \\ Received 4 April 2007 \\ Accepted 20 July 2007

\author{
Pauline Groussaud, Stephen J. Shankster, Mark S. Koylass \\ and Adrian M. Whatmore
} \\ Department of Statutory and Exotic Bacterial Diseases, Veterinary Laboratories Agency (VLA),
New Haw, Addlestone, Surrey KT15 3NB, UK \\ In order to investigate the genetic relationships within Brucella isolated from marine mammals, two genome-based typing methods, variable number of tandem repeats (VNTR) typing and multilocus sequence analysis (MLSA), were applied to a selection of 74 marine mammal isolates. All isolates were examined by VNTR and data were compared with multilocus sequencing data from a subset of 48 of these. Marine mammal brucellae are distinct from classically recognized species by these methods and appear to correspond to three major genetic groups, which reflect distinct preferred hosts. One group contains isolates predominantly found in pinnipeds (seals) and corresponds to the previously proposed species 'Brucella pinnipediae'. However, isolates corresponding to the previously proposed species 'Brucella cetaceae' fall into two distinct groups that appear to have different preferred cetacean hosts (porpoises and dolphins). Furthermore, these two groups appear less closely related to each other than either group is to ' $B$. pinnipediae' isolates. The groups identified by VNTR typing and MLSA are completely congruent. The relevance of these findings to current proposals to recognize two species of marine mammal Brucella is discussed.
}

\section{INTRODUCTION}

The genus Brucella contains Gram-negative, facultatively intracellular pathogens that can infect many species of animals of economic importance, such as cattle, sheep, goats and pigs (Cutler et al., 2005). Humans usually become infected through ingestion of unpasteurized dairy products or via occupational activities such as veterinary work, farming and butchery. Whilst animal brucellosis can present few symptoms and infection is often only recognized on abortion, in humans the disease causes flulike symptoms, including recurrent fever, chills, night sweats and nausea, as well as chronic fatigue and depression if the infection is not cleared early.

Brucella can also infect wild animals (Davis, 1990), and in recent years it has also been isolated from various marine mammal species. In 1994, two reports described the first isolation of Brucella from marine mammals. These strains originated from four common seals (Phoca vitulina), two

Abbreviations: IRS-PCR, infrequent restriction site PCR; MLSA, multilocus sequence analysis; SNP, single nucleotide polymorphism; ST, sequence type; VNTR, variable number of tandem repeats.

A table showing the VNTR typing profiles of the 74 isolates described in this study is available as supplementary data with the online version of this paper. harbour porpoises (Phocoena phocoena) and a common dolphin (Delphinus delphis) in Scotland (Ross et al., 1994), and a captive bottlenose dolphin (Tursiops truncatus) in California, USA (Ewalt et al., 1994). Since these initial isolations, marine mammal Brucella have been isolated from a range of animals, including Atlantic white-sided dolphins (Lagenorhynchus acutus), striped dolphins (Stenella coeuleoalba), grey seals (Halichoerus grypus), hooded seals (Cystophora cristata), European otters (Lutra lutra) (Foster et al., 1996), Pacific harbour seals (Phoca vitulina richardsi) (Garner et al., 1997), minke whales (Balaenoptera acutorostrata) (Clavareau et al., 1998), harp seals (Phoca groenlandica), ringed seals (Phoca hispida) (Forbes et al., 2000) and whitebeaked dolphins (Lagenorhynchus albirostris) (Foster et al., 2002).

Six Brucella species are currently recognized based on phenotypic traits and host preference: Brucella abortus (bovine), Brucella melitensis (ovine and caprine), Brucella suis (porcine), Brucella ovis (ovine), Brucella canis (canine) and Brucella neotomae (desert wood rat). Observations of very high levels of genetic relatedness within the group based on DNA-DNA hybridization experiments have led to the traditional view of Brucella taxonomy being challenged and to the group being described as monospecific (Verger et al., 1985). Practical considerations 
meant that this classification system never found widespread support and recently moves were made to return to the classical species designations (Osterman \& Moriyón, 2006). However, many molecular methods, including multilocus enzyme electrophoresis (MLEE) (Gandara et al., 2001), 16S rRNA sequencing (Gee et al., 2004), and amplified fragment length polymorphism (AFLP) (Whatmore et al., 2005) have supported the observation of an unusually high degree of genetic conservation within this group. This has recently been confirmed by multilocus sequencing, in which, as an extreme example, B. canis is separated from some $B$. suis strains by only one to two base changes in 4396 bp examined (Whatmore et al., 2007). The results presented in this paper need to be considered in this context of groups with minimal genetic separation being granted species status based on differences in phenotype and host specificity.

While the impact of Brucella infection on marine mammals is unclear, there is increasing interest in these bacteria both as potential animal pathogens and as agents of zoonotic infection of man (Brew et al., 1999; Sohn et al., 2003; McDonald et al., 2006). Although first identified in 1994, Brucella isolates from marine mammals still have to be formally named, reflecting the ongoing debate about the genetic relationships within this group and how these relationships relate to the proposed species designations (Osterman \& Moriyón, 2006). It was first suggested that the group should consist of a single new species, 'Brucella maris' (Jahans et al., 1997), which could be further subdivided into three different biovars. According to this scheme, biovar 1 comprised seal and otter isolates, biovar 2 comprised dolphin and porpoise isolates, while biovar 3 consisted of a single Californian bottlenose dolphin isolate (Ewalt et al., 1994). Later, Cloeckaert et al. (2001) argued that on the basis of DNA polymorphism at the omp2 locus and the classical criterion of preferential host, two species should be recognized with the suggested names 'Brucella pinnipediae' (seal isolates) and 'Brucella cetaceae' (dolphin and porpoise isolates). The authors noted that these two species groups correspond to the biovar 1 and 2 groupings described by Jahans et al. (1997). However, they proposed that the biovar 3 isolate represented a serotype rather than a third biovar, although they did not examine this isolate in their study. Later, a study from the same group, based on infrequent restriction site PCR (IRS-PCR), was reported to support their proposed classification (Cloeckaert et al., 2003).

In the following study, we aim to clarify genetic relationships within the marine mammal Brucella by applying two recently developed multilocus typing techniques, namely variable number of tandem repeats (VNTR) typing and multilocus sequencing, to a large collection of isolates obtained from a variety of marine mammal species. Genetic relationships within the group are examined in relation to mammalian host of origin and the implications of these findings to the ongoing taxonomic debate surrounding these isolates are discussed.

\section{METHODS}

Isolates and template preparation. All 74 isolates examined in this study were cultured at $37{ }^{\circ} \mathrm{C}$ with $10 \% \mathrm{CO}_{2}$ in Brodie and Sinton's broth, then subcultured onto serum dextrose agar for up to a month. The template for molecular assays consisted of either DNA prepared by classical phenol/chloroform extraction or methanol extracts prepared as described previously (Whatmore et al., 2005). Information describing the geographical origin of isolates and the mammalian host of origin is provided in Fig. 3.

Multilocus sequencing. Multilocus sequence analysis (MLSA) examining nine distinct genetic fragments was carried out as previously outlined by Whatmore et al. (2007) in a paper that describes the development of the multilocus sequencing scheme. Many of the profiles were originally mentioned in that paper, but these and additional isolates are examined in greater depth here in the context of relationships within the marine mammal Brucella and to provide a comparison with clustering obtained by VNTR-based approaches. This analysis includes 43 of the 45 strains originally described (the remaining two have not been examined by VNTR typing and were therefore excluded) and five additional isolates not described in Whatmore et al. (2007).

VNTR typing. A VNTR typing scheme that indexes diversity at 21 distinct loci was applied to all 74 isolates examined in this study, and was performed as described previously, with minor modifications to some primer concentrations (Whatmore et al., 2006). The raw VNTR data are available in Supplementary Table S1. Thirteen of the 74 isolates tested by VNTR showed a mixed profile at one or more loci previously shown to have a high diversity index (Whatmore et al., 2006). All but one of these mixed profiles represented a single step change in the number of repeats. The duplicates are labelled a, b, etc., in Supplementary Table S1, and only one profile from each strain (that labelled a) was included in Fig. 3. As the changes are very minor, all variant profiles from a single strain cluster closely together, and thus the inclusion of only a single variant does not alter the overall conclusions.

Relationships with Brucella species isolated from terrestrial mammals based on VNTR profiles were determined by using the neighbourjoining tree algorithm in the PAUP4.0 beta version (Sinauer Associates), as described previously (Whatmore et al., 2006). A tree previously constructed on the basis of 105 isolates representing all terrestrial species and biovars (Whatmore et al., 2006) was reconstructed with the addition of 10 marine mammal strains to examine the relationship of these isolates to other Brucella species. The relationships among all 74 marine mammal isolates were examined by cluster analysis using the categorical coefficient (tolerance $=0$, no fuzzy logic) and UPGMA implemented in BioNumerics version 4.60 (Applied Maths). The categorical coefficient treats each different value as a different state, implying that character sets are unordered: the same weight is therefore given to large or small numbers of differences.

\section{RESULTS AND DISCUSSION}

\section{Multilocus sequencing}

We recently published a multilocus sequencing scheme that examined the sequences of nine independent fragments of the Brucella genome (Whatmore et al., 2007). Based on examination of 45 marine mammal isolates in conjunction with 115 isolates representing all known Brucella species and biovars, the marine mammal isolates were found to 
constitute a distinct genetic cluster consisting of five sequence types (STs) labelled ST23 to ST27. This analysis supported the view that marine mammal strains constitute one or more novel species within the genus Brucella. The relationships between the five STs and the species of marine mammal from which the corresponding isolates originated are detailed in Table 1. ST24 and ST25 are the most closely related STs among the marine strains of Brucella, differing by one nucleotide polymorphism only (see Fig. 1). Both these STs appear to be predominantly associated with seals ( $84 \%$ of isolates). Isolates belonging to ST23 and ST26 are more distantly related to the sealassociated ST24 and ST25, and also display distinct apparent preferred hosts. Thus, ST26 was isolated from dolphin species only, while ST23 was predominantly associated with porpoises ( $74 \%$ of isolates). The remaining ST, ST27, was only isolated once and although this isolate was obtained from a bottlenose dolphin, it appeared to be more closely related to the seal STs than to those associated with porpoises and dolphins (two or three changes relative to seal STs as opposed to four or five changes relative to dolphin and porpoise STs; see Fig. 1).

\section{VNTR: relationship to existing Brucella species}

A VNTR scheme examining 21 markers with repeat units of $5 \mathrm{bp}$ or more was applied to all 74 Brucella isolates obtained from marine mammals. This scheme includes markers with a range of apparent evolutionary speeds and thus has been shown to be useful both in providing a phylogenetic signal and in allowing local epidemiological traceback (Whatmore et al., 2006). Fig. 2 shows the relationship of 10 marine mammal isolates to 105 isolates originally included in the study of Whatmore et al. (2006) and representing all known classical Brucella species and biovars. The marine mammal strains fall into three groups clearly distinct from previously described groups of Brucella isolated from terrestrial mammals. These three groups correspond to the major groups already described by MLSA. These findings are consistent with VNTR

\begin{tabular}{|l|c|c|c|c|c|}
\hline & ST24 & ST25 & ST27 & ST23 & ST26 \\
\hline ST24 & & 1 & 3 & 4 & 3 \\
\hline ST25 & & & 2 & 3 & 2 \\
\hline ST27 & & & & 5 & 4 \\
\hline ST23 & & & & & 5 \\
\hline ST26 & & & & & \\
\hline
\end{tabular}

Fig. 1. Number of variable nucleotide sites among marine mammal STs as determined by multilocus sequencing.

analysis performed using an alternative scheme (Le Fleche et al., 2006) in which three marine mammal strains were included in a maximum-parsimony analysis using 71 markers. This analysis also suggested that there are three distinct genetic groups of marine mammal Brucella, one corresponding to ' $B$. pinnipediae' (seals) and two groups corresponding to 'B. cetaceae' (dolphins and porpoises).

\section{VNTR: relationships among Brucella isolates from marine mammals}

In order to examine genetic relationships within the group, a dendrogram was constructed based on VNTR profiles for all 74 isolates examined in this study (Fig. 3). The MLSA results for the 48 isolates upon which this method was performed are superimposed on this figure. Three major and well-separated groups, congruent with those identified by MLSA, were identified by VNTR typing. The first of these groups (cluster A), consisting of 17 isolates obtained exclusively from dolphin species, corresponded to ST26. The second (cluster B) consisted of 37 isolates, $73 \%$ of which were isolated from porpoises. This group was congruent with ST23. The third major group (cluster C) contained 20 isolates, $80 \%$ of which were isolated from various seal species. This group fell into three subclusters (subclusters C1-C3). Two of these subclusters corresponded to ST24 (subcluster C1) and ST25 (subcluster C2),

Table 1. Summary of findings based on multilocus sequencing of 48 marine mammal Brucella

\begin{tabular}{|c|c|c|c|c|c|c|c|c|c|c|c|}
\hline \multirow{3}{*}{ ST } & \multicolumn{2}{|l|}{ Multilocus sequence profile ${ }^{\star}$} & 1 & 1 & 1 & 1 & 2 & 3 & 3 & $\begin{array}{l}\text { Number of } \\
\text { isolates }\end{array}$ & $\begin{array}{c}\text { Marine mammal species of } \\
\text { origin }\end{array}$ \\
\hline & & 9 & 9 & 5 & 5 & 1 & 9 & 4 & 1 & & \\
\hline & & 6 & 7 & 2 & 4 & 8 & 6 & 5 & 0 & & \\
\hline ST27 & 124311521 & $\mathrm{C}$ & G & G & G & A & A & G & $\mathrm{C}$ & 1 & 1 Dolphin \\
\hline ST23 & 148412521 & $\mathrm{~T}$ & G & A & A & G & G & G & $\mathrm{C}$ & 19 & 14 Porpoise, 2 seal, 3 dolphin \\
\hline ST26 & 124212671 & $\mathrm{C}$ & G & G & G & G & G & A & $\mathrm{T}$ & 9 & 9 Dolphin \\
\hline
\end{tabular}

${ }^{*}$ Multilocus sequencing profile $=g a p$, aroA, $g l k, d n a K, \operatorname{gyrB}, \operatorname{trp} E, \operatorname{cobQ}$, omp25, int-hyp.

$\dagger$ Location of SNPs is as follows: $696=\operatorname{aroA}, 1297$ and $1352=g l k, 1654$ and $1718=d n a K, 2796=\operatorname{trpE}, 3445=\operatorname{cobQ}, 3810=o m p 25$. 


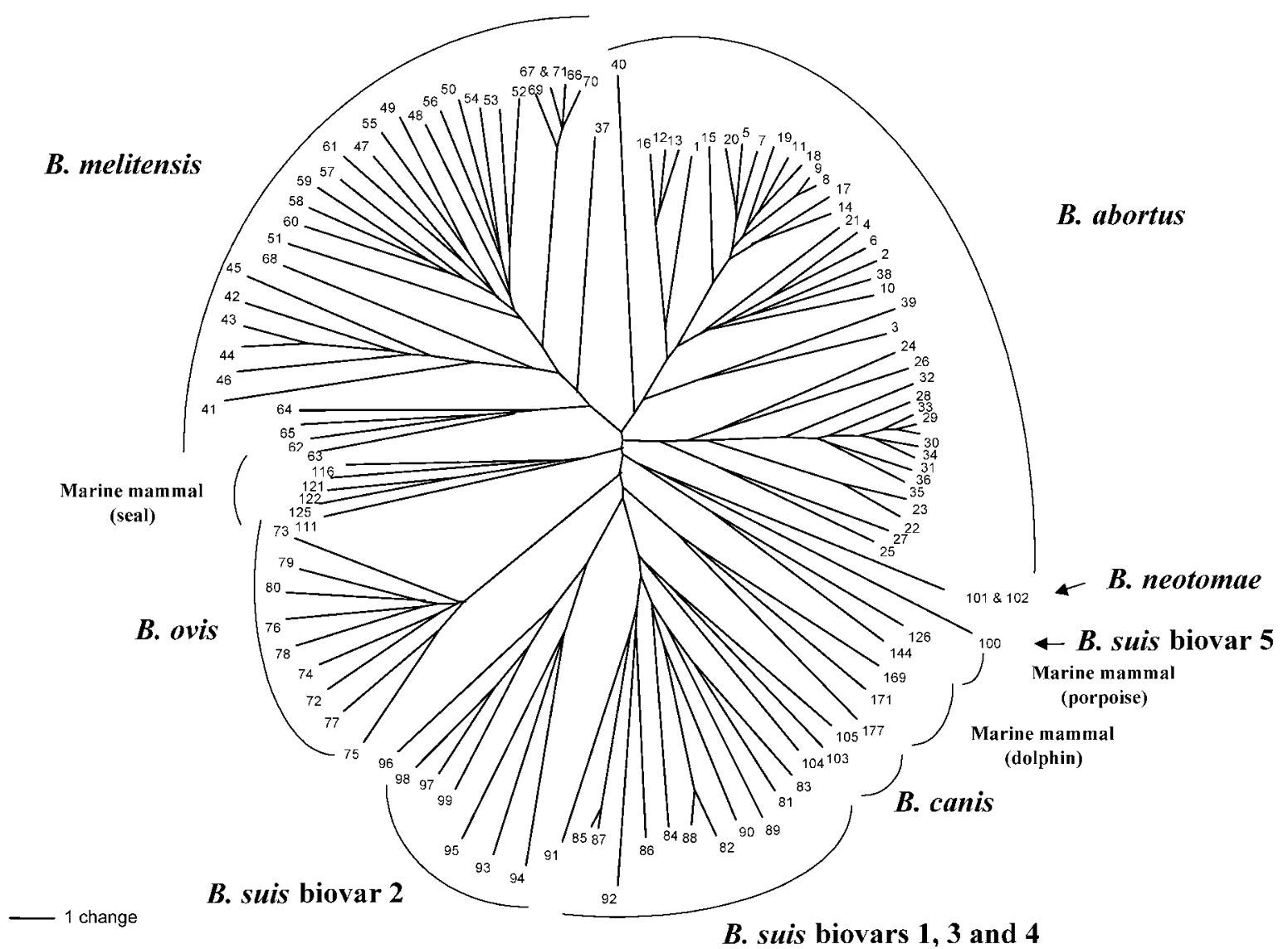

Fig. 2. Neighbour-joining reconstruction of relationships among 105 isolates representing all terrestrial Brucella species and 10 marine mammal Brucella isolates. Strain identities of the 105 isolates are as previously presented in Whatmore et al. (2006), while the identities of the marine mammal strains are included in Supplementary Table S1.

while the third consisted of ST27 and isolates of ST25 obtained from hooded seals (subcluster C3). It is notable that one of the cetacean groups (corresponding to ST23/ cluster B) appeared more closely related to the pinniped cluster (corresponding to ST24 and ST25/cluster C) than to the second cetacean cluster (ST26/cluster A). This is absolutely consistent with multilocus sequencing, in which ST23 differs from ST24 and ST25 by four and three single nucleotide polymorphisms (SNPs), respectively, and from ST26 by five SNPs.

To summarize, this analysis confirms that there are three major genetic groups in the Brucella population examined and facilitates understanding of the genetic structure of the group in relation to previously suggested taxonomic groupings. The clusters generally correspond to isolation from a predominant host group; thus one group, containing mainly seal isolates, corresponds to isolates previously described as ' $B$. pinnipediae'. However, isolates previously described as the ' $B$. cetaceae' group fall into two very distinct genetic clusters that correspond to isolates exclusively seen in dolphins and a group that is strongly associated with porpoises. The MLSA results superimposed onto the VNTR-based analysis show that groupings based on this approach are entirely congruent with those described by VNTR typing. Thus, the closely related ST24 and ST25 correspond to the seal group, while ST23 and ST26 correspond to the porpoise and dolphin groups, respectively. The isolate representing the remaining ST, ST27, appears rather distinct from any of these three groups, particularly by MLSA. The isolate representing this ST corresponds to the bottlenose dolphin isolate identified

Fig. 3. Relationships between 74 marine mammal Brucella isolates based on 21-locus VNTR analysis. Multilocus sequence types are superimposed as ST23 $(\bullet)$, ST $24(\star)$, ST25 $(\diamond)$, ST26 $(\odot)$ and ST27 $(\boldsymbol{\square})$. Host species abbreviations are as follows: hb, harbour; b, bottlenose; c, common; aws, Atlantic white-sided; g, grey; hd, hooded; e, European; s, striped; m, minke. In the case of UK isolates the year represents the year of isolation, while for overseas isolates it represents the year of receipt at the VLA. Clusters and subclusters are annotated as described in the text. 


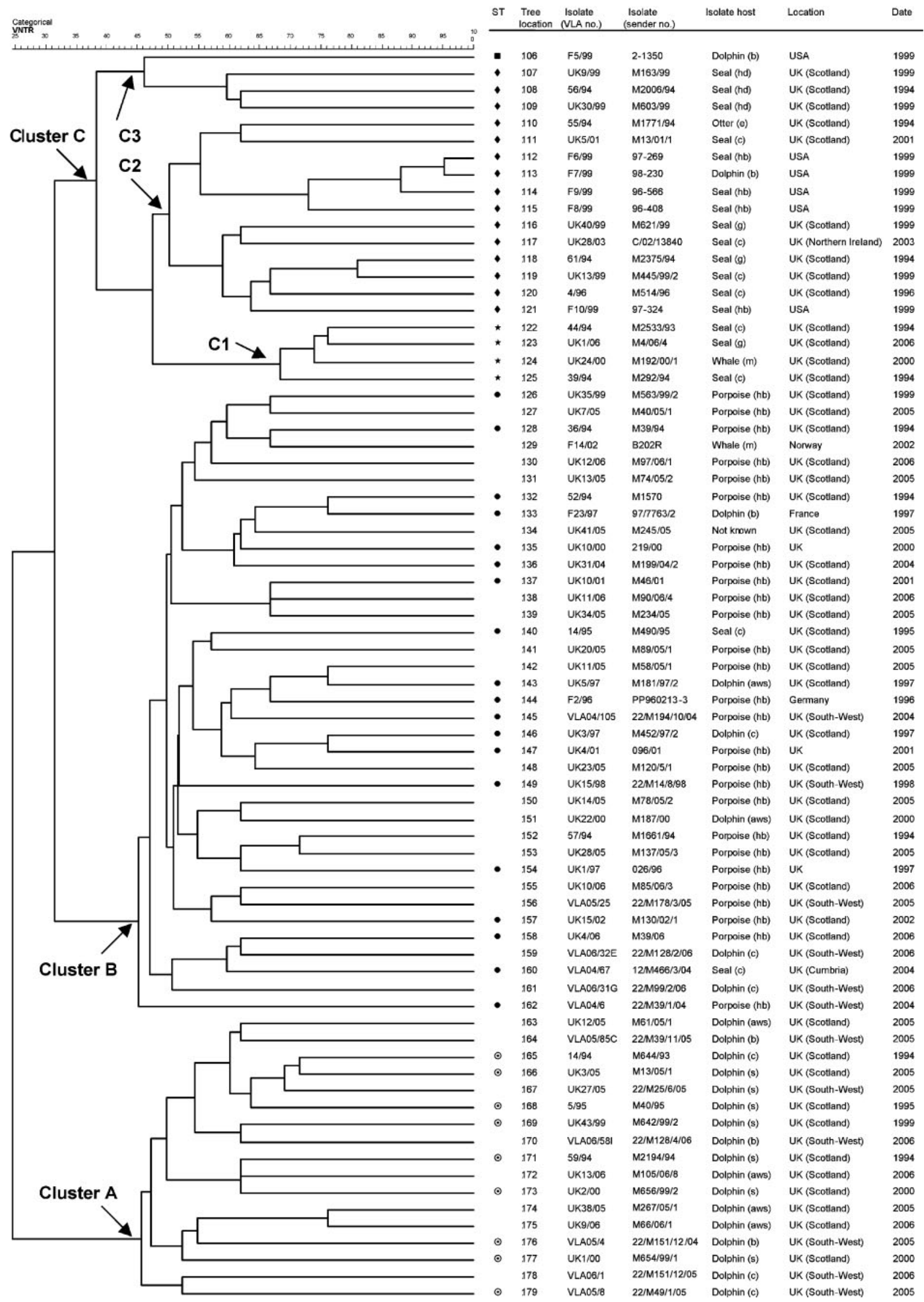


by Ewalt et al. (1994) and identified as ' $B$. maris' biovar 3 by Jahans et al. (1997). As we have only identified one isolate of this ST in marine mammals, it may be less common or absent in European waters, and identification of the true host range of this ST requires further isolations from the Pacific regions. Interestingly, the only other isolate of ST27 reported to date is from a human infection in New Zealand (McDonald et al., 2006), indicating that further study to identify the natural host and zoonotic potential of this ST would be valuable.

\section{Implications for Brucella classification}

Based on data presented here the subdivision of marine mammal isolates into multiple species could be justified on genetic grounds, despite their overall rather close relationship. There is clearly precedent for this in Brucella taxonomy. The STs representing the three major groups of marine Brucella strains are markedly less closely related to each other than some $B$. suis isolates and $B$. canis are to one another (Whatmore et al., 2007), yet these organisms are recognized as separate species due to the difference in host preference. However, the classification of the group into two species, ' $B$. pinnipediae' and 'B. cetaceae', appears inconsistent with the genetic relationships observed within the group in this study. The 'B. pinnipediae' group, consisting of isolates predominantly from seals, appears to be a valid genetic group. However, the isolates proposed as ' $B$. cetaceae' clearly consist of two well-separated genetic groups on the basis of both MLSA and VNTR. Moreover, these two groups appear less closely related to each other than either is to the ' $B$. pinnipediae' group (Fig. 1). We have further confirmed this by sequencing fragments of eight additional housekeeping genes from all isolates. The results of this further analysis are absolutely consistent with that based on the nine-locus MLSA in upholding the genetic relationships described here (data not shown). Furthermore, the two cetacean groups also fulfil one of the classical criteria for identification of Brucella species in that the data presented here suggest that they have different preferred hosts. Based on the data presented here, and given the apparent inconsistency with the proposed two species ('B. pinnipediae' and 'B. cetaceae'), there appear to be two possible alternative scenarios for classifying the marine mammal Brucella. The first would be the identification of a single marine mammal Brucella species that could then be further subdivided, perhaps into 'ecotypes', corresponding to the groups described here. The second would be the assignment of the cetacean isolates into two species according to host preference and genetic relationships, rather than into a single species ('B. cetaceae') as has been suggested, resulting in the identification of three species of Brucella isolated from marine mammals.

Although this division into three genetic groups with distinct host preferences was not noted by previous workers who suggested the division into 'B. pinnipediae' and 'B. cetaceae', this may reflect the small number of strains examined in these studies and the fact that the methods used do not give a direct measure of the genetic relationships between groups (Cloeckaert et al., 2001, 2003). Indeed, by specific PCRs based on IRS-PCR, three distinct genetic groups that correspond to the three identified in this study have been reported (Cloeckaert et al., 2003), although the distinct host preferences apparent in our study were not observed, as only four dolphin isolates corresponding to our ST26 were included in the study. Review of the literature shows that the division into three groups is supported by additional molecular methods. Jensen et al. (1999) showed using PFGE that dolphin isolates can be discriminated from both seal and porpoise isolates. Furthermore, the porpoise and seal groups appear more closely related to each other than they are to the dolphin group, as noted in this study. Physical mapping studies are also reported to divide the marine mammal Brucella into the same three groups, whereby seal and porpoise isolates are more closely related to each other than to dolphin isolates (Boschiroli et al., 2001; Bourg et al., 2007).

\section{Summary}

It is widely acknowledged that marine mammal Brucella form a group separate from classically recognized Brucella, as first demonstrated by Ross et al. (1994) and Ewalt et al. (1994). This is supported by both MLSA- and VNTR-based typing. It is clear from this study, and from others discussed above, that at least three major groups of marine mammal Brucella can be defined by molecular approaches. The relevance of this to taxonomy and species definition needs to be discussed. While taxonomy is not driven by genotype alone, it should ideally reflect both genetic and phenotypic divisions, and the division into two potential species (' $B$. pinnipediae' and 'B. cetaceae') appears inconsistent with the genetic relationships observed in this study. Following such a scheme, some isolates of ' $B$. cetaceae' would be more closely related to ' $B$. pinnipediae' than to other isolates of ' $B$. cetaceae'. The data presented here appear more compatible with proposals outlined by Corbel \& Banai (2005) in the latest Bergey's Manual of Systematic Bacteriology, in which the possibility of identification of three novel species, 'Brucella phocae' (seals), 'Brucella phoecoenae' (porpoises) and 'Brucella delphini' (dolphins), was raised.

\section{ACKNOWLEDGEMENTS}

We gratefully acknowledge colleagues who have provided strains included in this study, particularly Geoff Foster [Scottish Agricultural College (SAC), Inverness] and Nick Davison (VLA Truro). Research within the group is supported by the UK Department for Environment, Food and Rural Affairs (Defra).

\section{REFERENCES}

Boschiroli, M. L., Foulongne, V. \& O'Callaghan, D. (2001). Brucellosis: a worldwide zoonosis. Curr Opin Microbiol 4, 58-64.

Bourg, G., O'Callaghan, D. \& Boschiroli, M. L. (2007). The genomic structure of Brucella strains isolated from marine mammals gives clues to evolutionary history within the genus. Vet Microbiol (in 
press).http://dx.doi.org. doi: 10.1016/j.vetmic.2007.06.002.10.1016/ j.vetmic.2007.06.002

Brew, S. D., Perrett, L. L., Stack, J. A., MacMillan, A. P. \& Staunton, N. J. (1999). Human exposure to Brucella recovered from a sea mammal. Vet Rec 144, 483.

Clavareau, C., Wellemans, V., Walravens, K., Tryland, M., Verger, J. M., Grayon, M., Cloeckaert, A., Letesson, J. J. \& Godfroid, J. (1998). Phenotypic and molecular characterization of a Brucella strain isolated from a minke whale (Balaenoptera acutorostrata). Microbiology 144, 3267-3273.

Cloeckaert, A., Verger, J. M., Grayon, M., Paquet, J. Y., Garin-Bastuji, B., Foster, G. \& Godfroid, J. (2001). Classification of Brucella spp. isolated from marine mammals by DNA polymorphism at the omp2 locus. Microbes Infect 3, 729-738.

Cloeckaert, A., Grayon, M., Grepinet, O. \& Boumedine, K. S. (2003). Classification of Brucella strains isolated from marine mammals by infrequent restriction site-PCR and development of specific PCR identification tests. Microbes Infect 5, 593-602.

Corbel, M. J. \& Banai, M. (2005). Genus I. Brucella Meyer and Shaw 1920, 173AL. In Bergey's Manual of Systematic Bacteriology, vol. 2, pp. 370-386. Edited by D. J. Brenner, N. R. Krieg \& J. T. Staley. New York: Springer.

Cutler, S. J., Whatmore, A. M. \& Commander, N. J. (2005). Brucellosis - new aspects of an old disease. J Appl Microbiol 98, 1270-1281.

Davis, D. S. (1990). Brucellosis in wildlife. In Animal Brucellosis, pp. 321-34. Edited by K. Nielsen \& J. R. Duncan. Boca Raton, FL: CRC Press.

Ewalt, D. R., Payeur, J. B., Martin, B. M., Cummins, D. R. \& Miller, W. G. (1994). Characteristics of a Brucella species from a bottlenose dolphin (Tursiops truncatus). J Vet Diagn Invest 6, 448-452.

Forbes, L. B., Nielsen, O., Measures, L. \& Ewalt, D. R. (2000). Brucellosis in ringed seals and harp seals from Canada. J Wildl Dis 36, 595-598.

Foster, G., Jahans, K. L., Reid, R. J. \& Ross, H. M. (1996). Isolation of Brucella species from cetaceans, seals and an otter. Vet Rec 138, 583-586.

Foster, G., MacMillan, A. P., Godfroid, J., Howie, F., Ross, H. M., Cloeckaert, A., Reid, R. J., Brew, S. \& Patterson, I. A. (2002). A review of Brucella sp. infection of sea mammals with particular emphasis on isolates from Scotland. Vet Microbiol 90, 563-580.

Gandara, B., Merino, A. L., Rogel, M. A. \& Martinez-Romero, E. (2001). Limited genetic diversity of Brucella spp. J Clin Microbiol 39, 235-240.

Garner, M. M., Lambourn, D. M., Jeffries, S. J., Hall, P. B., Rhyan, J. C., Ewalt, D. R., Polzin, L. M. \& Cheville, N. F. (1997). Evidence of Brucella infection in Parafilaroides lungworms in a Pacific harbor seal (Phoca vitulina richardsi). J Vet Diagn Invest 9, 298-303.

Gee, J. E., De, B. K., Levett, P. N., Whitney, A. M., Novak, R. T. \& Popovic, T. (2004). Use of $16 \mathrm{~S}$ rRNA gene sequencing for rapid confirmatory identification of Brucella isolates. J Clin Microbiol 42, 3649-3654.

Jahans, K. L., Foster, G. \& Broughton, E. S. (1997). The characterisation of Brucella strains isolated from marine mammals. Vet Microbiol 57, 373-382.

Jensen, A. E., Cheville, N. F., Thoen, C. O., MacMillan, A. P. \& Miller, W. G. (1999). Genomic fingerprinting and development of a dendrogram for Brucella spp. isolated from seals, porpoises, and dolphins. J Vet Diagn Invest 11, 152-157.

Le Fleche, P., Jacques, I., Grayon, M., Al Dahouk, S., Bouchon, P., Denoeud, F., Nockler, K., Neubauer, H., Guilloteau, L. A. \& Vergnaud, G. (2006). Evaluation and selection of tandem repeat loci for a Brucella MLVA typing assay. BMC Microbiol 6, 9.

McDonald, W. L., Jamaludin, R., Mackereth, G., Hansen, M., Humphrey, S., Short, P., Taylor, T., Swingler, J., Dawson, C. E. \& other authors (2006). Characterization of a Brucella sp. strain as a marine-mammal type despite isolation from a patient with spinal osteomyelitis in New Zealand. J Clin Microbiol 44, 4363-4370.

Osterman, B. \& Moriyón, I. (2006). International Committee on Systematics of Prokaryotes. Subcommittee on the Taxonomy of Brucella. Report of the Meeting, 17 September 2003, Pamplona, Spain. Int J Syst Evol Microbiol 56, 1173-1175.

Ross, H. M., Foster, G., Reid, R. J., Jahans, K. L. \& MacMillan, A. P. (1994). Brucella species infection in sea-mammals. Vet Rec 134, 359.

Sohn, A. H., Probert, W. S., Glaser, C. A., Gupta, N., Bollen, A. W., Wong, J. D., Grace, E. M. \& McDonald, W. C. (2003). Human neurobrucellosis with intracerebral granuloma caused by a marine mammal Brucella spp. Emerg Infect Dis 9, 485-488.

Verger, J. M., Grimont, F., Grimont, P. A. D. \& Grayon, M. (1985). Brucella, a monospecific genus as shown by deoxyribonucleic acid hybridization. Int J Syst Bacteriol 35, 292-295.

Whatmore, A. M., Murphy, T. J., Shankster, S., Young, E., Cutler, S. J. \& Macmillan, A. P. (2005). Use of amplified fragment length polymorphism to identify and type Brucella isolates of medical and veterinary interest. J Clin Microbiol 43, 761-769.

Whatmore, A. M., Shankster, S. J., Perrett, L. L., Murphy, T. J., Brew, S. D., Thirlwall, R. E., Cutler, S. J. \& Macmillan, A. P. (2006). Identification and characterization of variable-number tandem-repeat markers for typing of Brucella spp. J Clin Microbiol 44, 1982-1993.

Whatmore, A. M., Perrett, L. L. \& Macmillan, A. P. (2007). Characterisation of the genetic diversity of Brucella by multilocus sequencing. BMC Microbiol 7, 34. 